\title{
Monitoring geomagnetic signals of groundwater movement using multiple underground SQUID magnetometers
}

\author{
S. Henry ${ }^{1}$, E. Pozzo di Borgo ${ }^{2}$, C. Danquigny ${ }^{3}$, A. Cavaillou ${ }^{3}$, A. Cottle ${ }^{1}$, S. Gaffet $^{3}$ and M. Pipe ${ }^{1}$ \\ ${ }^{1}$ University of Oxford, Department of Physics, Denys Wilkinson Building, Keble Road, Oxford \\ OX1 3RH, UK \\ ${ }^{2}$ UAPV, UMR1114 EMMAH, 84914 Avignon, France; INRA, UMR1114 EMMAH, \\ 84914 Avignon, France \\ ${ }^{3}$ Université de Nice Sophia-Antipolis, Observatoire de la Côte d'Azur, Laboratoire Souterrain à Bas \\ Bruit de Rustrel-Pays d'Apt (LSBB), La Grande Combe, 84400 Rustrel, France
}

\begin{abstract}
Groundwater can influence the geomagnetic field measured underground in at least two key ways. The water levels in rock will determine its electrical conductivity, and thus change the magnitude of the telluric currents induced in the rock by changing magnetic fields generated in the ionosphere. This can be studied by using multiple magnetometers at different underground locations. Secondly the flow of water through rock will generate a small magnetic signal, of unknown magnitude, through the electrokinetic effect. SQUID magnetometry has the potential to allow passive studies of groundwater changes in complex systems such as karst. We have monitored geomagnetic signals using two SQUID magnetometers at the LSBB underground laboratory, and set an initial limit on the magnitude of the electrokinetic signal. We now plan to carry out a longer term measurement using three SQUID systems as well as fluxgate sensors to track changes in the gradient of the magnetic field across the underground complex.
\end{abstract}

\section{Introduction}

The changing magnetic field recorded at a given point in the Earth's crust is the sum of the fields produced by a number of different processes [1]. The geodynamo inside our planet provides the dominant contribution $(\sim 40 \mu \mathrm{T}$ at the latitude of LSBB), which changes only very slowly over a timescale of years. There are fluctuations on shorter timescales. Daily fluctuations in the magnetic field are produced by electrical currents in the ionosphere, driven by solar energy. This changing magnetic field will induce secondary currents in the Earth's crust (telluric currents), which will in turn generate a magnetic field [2]. The movement of water in the crust can generate a magnetic signal by the electrokinetic effect, and potentially other mechanisms [3].

In most areas with any significant level of human activity, anthropogenic magnetic noise will swamp natural fluctuations. The low noise environment at the LSBB (Laboratoire Souterrain à Bas Bruit) facility provides an ideal site to study geomagnetic phenomena [4]. In this paper we report on measurements carried out using two SQUID (Superconducting Quantum Interference Device)

This is an Open Access article distributed under the terms of the Creative Commons Attribution License 4.0, which permits unrestricted use, distribution, and reproduction in any medium, provided the original work is properly cited. 


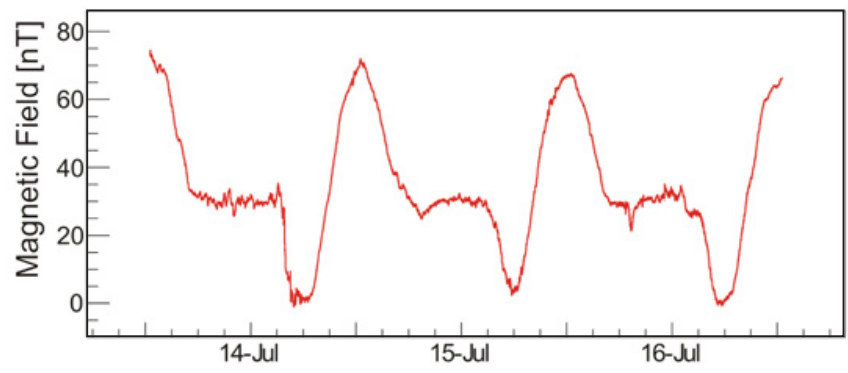

Figure 1. Geomagnetic field variations (EW component) recorded with a SQUID system at LSBB over a 72 hour period [6]. The SQUID is not an absolute magnetometer so the vertical axis has an arbitrary offset. This magnetic signal is primarily due to currents in the ionosphere. This changing magnetic field induces telluric currents in the earth's crust, which also contribute to the measured signal at a low level.
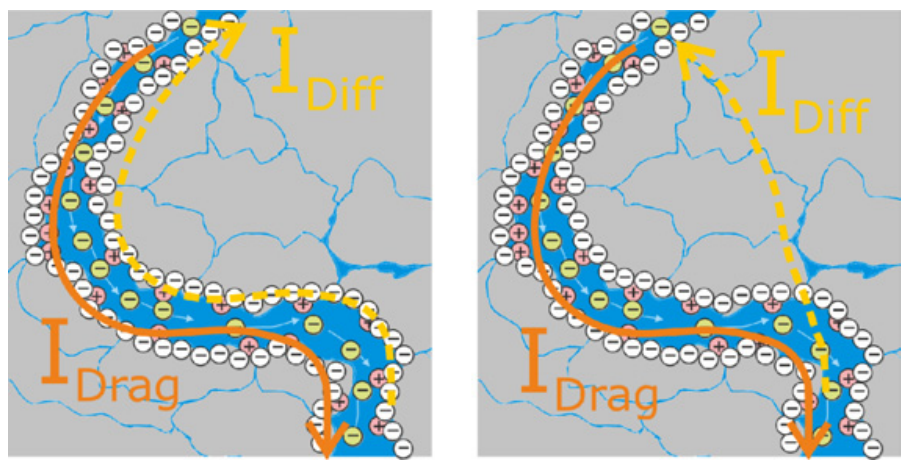

Figure 2. Currents produced by the electrokinetic effect due to the flow of water through rock. Left: if the drag and diffusion current are balanced there will be no net magnetic field. Right: if the diffusion current returns along a different path this will produce a magnetic field.

magnetometers. SQUIDs exploit the quantum properties of superconducting loops with Josephson Junctions to detect tiny changes in magnetic flux, allowing them to track geomagnetic fluctuations at a sub-picotesla level [5]. The aim of this project was to see if the improved resolution of SQUIDs compared to fluxgate magnetometers, with a resolution $\sim 0.1 \mathrm{nT}$, could detect weak magnetic signals below this magnitude.

In the following section, we give more details on expected geomagnetic signals. We then describe the simultaneous measurements of geomagnetic fluctuations carried out at two points in the underground laboratory, together with measurements of the rate of water flow through the tunnel wall. In the final section, we use this to give a limit on the magnitude of the signal produced by the electrokinetic effect, and discuss further measurement, currently in progress.

\section{Geomagnetic signals}

Figure 1 shows the magnetic field recorded by a SQUID system at the LSBB laboratory over a three day period. The diurnal pattern is primarily of ionospheric origin, but there is also a contribution from telluric currents which cannot be separated.

In addition to these sources, there will also be magnetic fields produced by the flow of the water through the rock, produced by the electrokinetic effect. Figure 2 illustrates how this is produced. Due to the potential difference across the rock-water interface (the zeta potential [3]), positive ions 
in groundwater are drawn to the surface, whereas negative ions are dragged along with the flow. The resulting drag current is balanced by the diffusion current to ensure charge conservation. If these two currents follow the same path (with opposite directions), then there is no net current or magnetic field. However in a realistic medium, the diffusion current will follow a different path as the path of lowest electrical resistance will vary from the path of minimum hydraulic resistance. This produces a current loop, and a resulting magnetic field [7].

Without detailed information about the surrounding rock, it is not possible to calculate the magnitude of this effect to any accuracy. We have carried out calculations for a range of models with different current distributions giving estimates of the magnetic signal at LSBB due to the infiltration of rainwater of between 0 and $1 \mathrm{nT}$. $^{1}$

The electrokinetic effect seems the most likely phenomenon to produce a magnetic signal proportional to the groundwater flow. But such a signal could conceivably be generated by other effects such as the deposition and impregnation of rocks with magnetic materials by fluids.

\section{Measurement at LSBB, October 2012}

To investigate the connection between the flow of groundwater and the geomagnetic field variations, we set up an experiment at LSBB using two magnetometers. A 3-axis SQUID system developed by Oxford University (originally for a neutron electric dipole moment experiment [8]) was installed $363 \mathrm{~m}$ below the surface at a point of monitored water flow. A second magnetometer was used as a reference to monitor the background fluctuations due to ionospheric currents. This was the [SQUID] ${ }^{2}$ instrument [9] permanently installed in the LSBB Capsule - a very low noise location in a room with thick steel walls. Both systems were calibrated together using a $0.9 \mathrm{~m}$ diameter external coil. The calibration signals were calculated by numerically integrating the Biot-Savart law.

The metal walls of the Capsule gave rise to a frequency dependent shielding factor. Fluctuations above $40 \mathrm{~Hz}$ were substantially attenuated. The quasi-dc signals studies studied here were $\sim 1.35$ times larger at point $\mathrm{C}$ compares to those recorded in the Capsule.

The account for the magnetic shielding, as well as any small calibration differences between the sensors, or small different in the alignment of the three axes, a linear transformation was applied to the $\mathrm{X}, \mathrm{Y}, \mathrm{Z}$ magnetic field data recorded in the Capsule,

$$
S^{\prime}=A X+B Y+C Z+D .
$$

Calculating $\mathrm{A}, \mathrm{B}, \mathrm{C}$ and $\mathrm{D}$ to give the minimum difference between $S^{\prime}$ and the signal recorded at point $C$. The parameters are calculated over a reference period at a different time to the data used to search for a signal related to groundwater flow. This method is described in Ref. 6 where it was used to

${ }^{1}$ The current density produced through the EK effect by a pressure gradient $\nabla \mathrm{P}$ is given by

$$
\mathrm{J}=\frac{\phi \varepsilon \zeta}{\eta} \nabla \mathrm{P}
$$

Where $\varphi$ is the rock porosity, $\varepsilon$ is the permittivity of water, $\zeta$ is the zeta potential of the interface, and $\eta$ is the viscosity of the water. Taking typical values of $\varphi=10^{-2}, \varepsilon=80 \varepsilon_{0}, \zeta=30 \mathrm{mV}, \eta=10^{-3} \mathrm{~Pa} \mathrm{~s}$, and $\nabla \mathrm{P}=10^{4} \mathrm{~Pa} \cdot \mathrm{m}^{-1}$, we estimate a volume averaged drag current density of $\sim 2 \mu \mathrm{Am}^{-2}$.

To get a rough estimate of the magnetic signal expected due to groundwater infiltration, we calculate the magnetic signals expected due to a current distribution in a $3 \times 3 \mathrm{~km}$ region centred on the laboratory, with a downwards current following a random walk starting from $250 \mathrm{~m}$ grid points on the surface, (which ranged from 390 to $1060 \mathrm{~m}$ asl) to a flat plane below the surface $\left(200 \mathrm{~m}\right.$ asl). This was balanced by a uniform upwards current density $2 \mu \mathrm{Am}^{-2}$ from the surface. The magnetic field was calculated for 10,000 random paths giving answers for the magnetic field gradient between the two measurement points of between 0 and $1 \mathrm{nT}$. We stress that this simulation is not based on any physical model. The reality is likely to be different, but this is only intended to give a very rough idea of the magnitude of a possible signal. 


\section{E3S Web of Conferences}
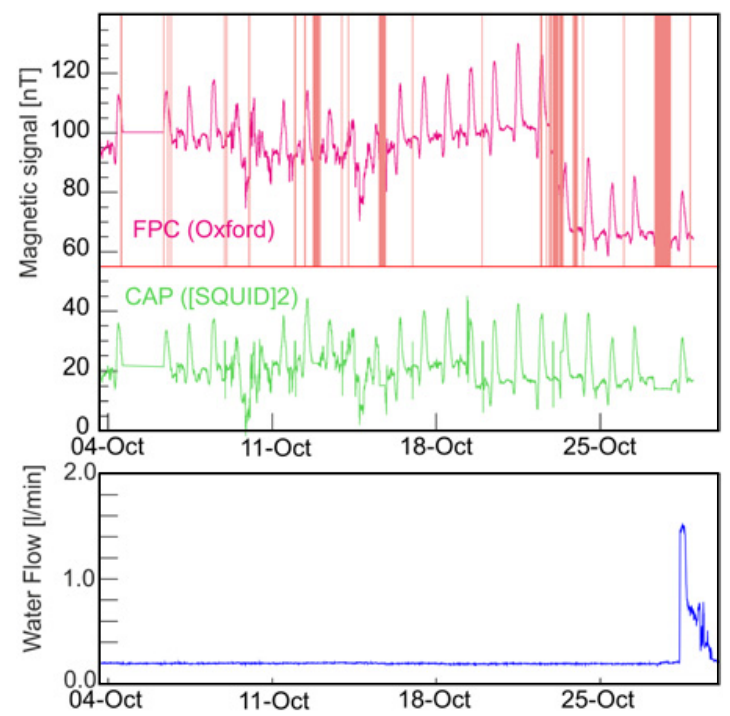

Figure 3. Magnetic signals recorded by SQUIDs at the water flow point C (FPC) and the Capsule (CAP) and the water flow rate. The red lines in the FPC plot indicate periods when the magnetic signal could not be accurately tracked due to rapidly changing fields or interference effects.

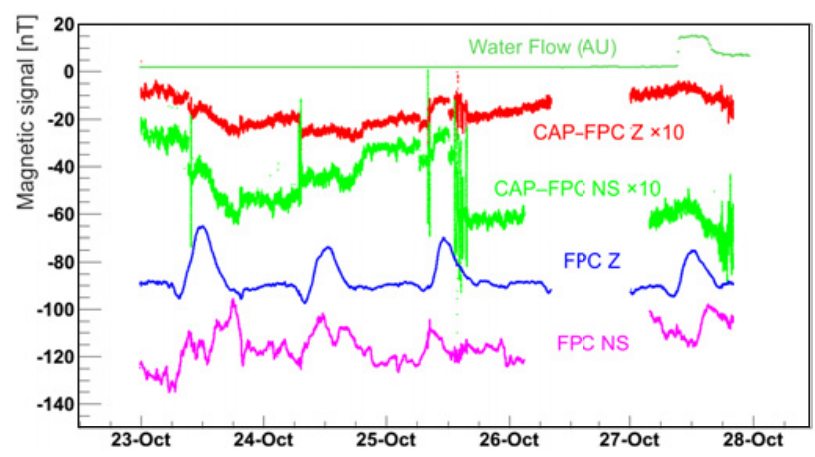

Figure 4. Magnetic signals recorded by SQUIDs at the time of the water flow anomaly on 27 October for the vertical (Z) and horizontal (NS) component. The plot shows the difference signal between the FPC magnetometer and the CAP reference (enlarged by a factor of 10). No significant magnetic signal is seen at the instance of the water flow spike. The abrupt anomalies seen on the NS difference signal can be attributed to the air pressure wave following the opening on the main tunnel door. The remaining difference is explained by the gradient of the telluric signals.

show that when the two SQUID systems were both located in the Capsule they tracked the same signal with an RMS difference of $<144$ pT over a 72 hour period.

Figure 3 shows the signals recorded by both magnetometers over a four week period in October 2012. These both show the same pattern of diurnal fluctuations and occasional larger disturbances due to magnetic storms. The water flow rate was constant for most of this month, but increased very rapidly on 27 October following a heavy rain storm.

Figure 4 shows the difference of the magnetic field at the flow point, and the rescaled CAP reference, for a five day period including the water flow anomaly on 27 October. The general fluctuations in the difference signal can be explained by the gradient of the field between these two locations, due to the 
local telluric currents and inhomogeneous magnetic shielding properties of the rock. The periodic spikes on the NS signal can be attributed to a pressure wave following the opening of the main tunnel door.

There is no significant change in the magnetic field at the time of the water flow anomaly. From the deviation of the background difference signal at this point we can say that any magnetic signal related to this must be below $0.26 \mathrm{nT}(\mathrm{NS})$ and $0.13 \mathrm{nT}(\mathrm{Z})$.

\section{Conclusions and future plans}

We present data from a preliminary investigation into the geomagnetic field fluctuations at the LSBB underground laboratory, and the link with groundwater flow. Simultaneous measurements with two 3-axis SQUID magnetometers have allowed us to establish that the magnitude of a magnetic signal correlated with the water flow (expected due to the electrokinetic effect) is less than $0.13 \mathrm{nT}$ (vertical component) and $0.26 \mathrm{nT}$ (horizontal components).

We are currently running a further investigation using 3-axis SQUID magnetometers at three locations in the LSBB complex. This will allow us to better map the gradient across the rock medium, in order to better study the magnetotelluric signals and the influence of groundwater on the geomagnetic field.

This project has been possible thanks to the generous support of INRA-UAPV-EMMAH; the Oxford-CNRS collaboration scheme; Hertford College, Oxford; and the Science and Technology Facilities Council (through the cryoEDM experiment). The authors would like to thank all the staff at LSBB for their assistance, and Prof Hans Kraus (Oxford) who developed the data acquisition hardware and software for the Oxford system.

\section{References}

[1] J. Jacobs, Geomagnetism (Academic Press, 1991).

[2] A. Chave, A. Jones, The Magnetotelluric Method. Theory and Practice (CUP, 2012).

[3] L. Jouniaux, T. Ishido, Int. J. Geophys. 286107 (2012).

[4] G. Wayand J. Phys Conf. Ser. 39, 157 (2006).

[5] J. Clarke, A. Braginski, The SQUID Handbook (Wiley VCH, 2004).

[6] S. Henry, E. Pozzo di Borgo, A. Cavaillou, Rev. Sci. Instrum. 84, 024501 (2013).

[7] T. Ishido, J. W. Pritchett, Geothermal Resource Council Transactions. 25, 645-649 (2001).

[8] S. Henry, H. Kraus, M. Malek, V. B. Mikhailika, G. Waysand, J. Instrum. 3: P11003 (2008).

[9] G. Waysand, J. Marfaing, E. Pozzo di Borgo E et al., Comptes Rendus Physique. 12, 192-202 (2011). 